\title{
Discrete Local Fairing of B-Spline Surfaces ${ }^{\star}$
}

\author{
Seok-Yong Hong, Chung-Seong Hong, Hyun-Chan Lee, and Koohyun Park \\ Department of Information and Industrial Engineering, \\ Hong-Ik University, Sangsu-dong 72-1, Mapo-gu, \\ Seoul, Republic of Korea (121-791) \\ sy_hong@orgio.net, pulnaeum@shinbiro.com, \\ hclee@wow.hongik.ac.kr, khpark@wow.hongik.ac.kr
}

\begin{abstract}
Many surfaces can be modeled by interpolating data points digitized from existing products. But the digitized data points could have measuring errors. To adjust the points, fairing is performed. We present an automatic local fairing algorithm using nonlinear programming. For the objective function of the algorithm, we derive discrete fairness metrics. The metrics are consisted of discrete principal curvatures. The discrete principal curvatures are calculated with the given data points.
\end{abstract}

\section{Introduction}

Reverse engineering is popular in product design. In reverse engineering, surfaces can be modeled by interpolating data points digitized from existing products. Designers can model new products by modifying the surfaces. But the digitized points could have noises. If the surfaces are constructed with such points, they have unwanted shapes. Fairing is necessary to adjust the points with noises.

Many existing fairing algorithms bring about good fairing results. However, excessive fairing of surface could have a problem. The problem is that the pattern of local shapes of an original surface is not preserved after fairing [3.5]. Thus, we present a new fairing algorithm, which performs iterative local fairing of data points of B-spline surfaces. As a result, it brings about fair surfaces and preserves the pattern of local shapes of an original surface. Because it performs fairing of data points, we adopted new discrete fairness metrics. The fairness metrics contain discrete principal curvatures. We derived the discrete principal curvatures from the data points.

\section{Discrete Fairness Metrics}

Fairness criteria are necessary to determine whether a surface is fair or unfair. The presented algorithm can use various fairness criteria such as flattening, rounding, and rolling. Designers can choose a fairness criterion suitable to design intents. Once a fairness criterion is selected, fairness of a surface must be measured numerically. The numerical measure of fairness is called fairness metric.

\footnotetext{
* This research was supported by Brain Korea 21 grant.
} 


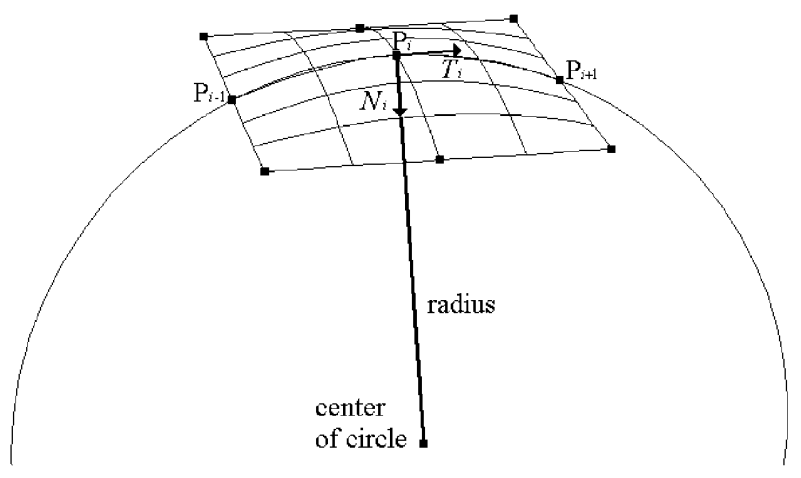

Fig. 1. Calculating discrete nomal curvatures with data points

We adopted the concept of derived surface to calculate discrete fairness metrics used in fairing data points [5]. A derived surface is consisted of geometric invariants such as curvature, radius of curvature, torsion, unit normal vector, unit tangent vector, and unit binormal vector. Once the derived surface for a fairness criterion is determined, we can derive a fairness metric by calculating the area of the derived surface. The fairness metric is used as the objective function of an optimization problem for fairing. If we minimize the objective function, the surface under consideration becomes fair. One of the fairness metrics we used is a rolling metric. If the rolling metric is minimized, an original surface tends to be made more cylindrical or conical. The rolling metric is shown in the equation (1). In the equation, $W$ is defined as $K+H^{2} . K, H, k_{1}$, and $k_{2}$ denote Gaussian curvature, mean curvature, and two principal curvatures, respectively. $s$ and $t$ are the parameters of the surface to be faired.

$$
\iint|W|\left[\left(k_{1} \frac{\partial W}{\partial t}\right)^{2}+\left(k_{2} \frac{\partial W}{\partial s}\right)^{2}+W^{2} K^{2}\right]^{1 / 2} d s d t
$$

To adopt the concept of the fairness metrics stated above in fairing data points, discrete fairness metrics must be derived. A discrete fairness metric is calculated with data points and it is consisted of discrete principal curvatures. To calculate the discrete principal curvatures at a data point, discrete normal curvatures at the data point must be derived. If data points are positioned in rectangular manner, a data point is surrounded by eight neighboring data points as shown in Fig. 1. Thus, discrete normal curvatures at the data point can be calculated in four directions, because they are calculated using the circles passing through three consecutive points containing the data point. The four discrete normal curvatures can be calculated using the original concept of normal curvature. The original normal curvature $\kappa_{n}$ at a point on a surface can be calculated as follows 11:

$$
\kappa_{n}=\kappa N \cdot n
$$


The four directional discrete normal curvatures at a data point can be calculated as follows:

First, discrete curvature at the data point is substituted for the curvature $\kappa_{n}$ in the equation (2). As shown in Fig. 1 with the given three consecutive points, $P_{i-1}, P_{i}$, and $P_{i+1}$, discrete curvature $\kappa_{i}$ at the point $P_{i}$ is calculated as the inverse of the radius of the circle passing through the three points. In addition, its first derivative can be calculated with discrete curvatures and the distances between the given points 2 .

Second, the discrete main normal vector $N_{i}$ at the same data point is substituted for the main normal vector $N$ of a curve on a surface. It can be calculated as the unit vector, of which direction is from the data point to the center of the circle passing through the three points used in calculating the discrete curvature at the data point.

Third, to approximate the unit normal vector $n$ of a surface, we calculate four directional unit tangent vectors with the data point and eight neighboring data points. In Fig. $1 T_{i}$ denotes one of the four unit tangent vectors. Each of the four vectors is tangent to the corresponding circle passing through the three consecutive points used for the discrete curvature. Then, we calculate two cross product vectors for two pairs of unit tangent vectors. One pair of the unit tangent vectors are of horizontal and vertical directions. The other pair of the vectors are the unit tangent vectors of diagonal directions. Finally, we can approximate the unit normal vector $n$ by calculating the average of the two cross product vectors.

Therefore, we can calculate four directional discrete normal curvatures at a data point using the derived discrete curvatures and normal vectors. Then we select a minimum discrete normal curvature and the other discrete normal curvature of orthogonal direction. The two curvatures are the discrete principal curvatures 4]. The discrete principal curvatures and the first derivatives of the discrete principal curvatures are used in calculating discrete fairness metrics.

\section{Discrete Fairing Algorithm}

The proposed discrete fairing algorithm performs iterative local fairing. For local fairing, a local fairness function is chosen as objective function. It evaluates fairness at a data point. Then, global fairness function is evaluated by accumulating all local fairness function values. It evaluates fairness for whole data points. The procedures of the algorithm are as follows:

Step 1. A data point set is given as input data.

Step 2. For a fairness metric, the value of the local fairness function for each data point and the value of the global fairness function are calculated.

Step 3. The point of which value of the local fairness function is the largest is selected.

Step 4. An optimization problem is formulated for improving fairness at the selected data point as follows. First, the local fairness function for the selected 
data point is used as the objective function of the optimization problem. Second, the free variables of the problem are the coordinates of the selected data point. Third, a constraint is set from the distance measure between the original data point and the modified data point. Then, new data point is calculated through the optimization process.

Step 5. If new value of the global fairness function is reduced, take the new point as the modified data point and go to step 2. If it is not reduced, the point of which value of the local fairness function is the next largest is selected and go to step 4 . However, if no more point exists to select, go to step 6 .

Step 6. A new B-spline surface is constructed by interpolating the modified data point set.

\section{Experimental Results}

We tested the proposed discrete fairing algorithm with an example data point set. Fig. 2 shows the results of fairing with a rolling metric. The figure contains an original surface, the surface faired with the discrete fairing algorithm, and the surface faired with an analytic fairing algorithm. The original surface is constructed by interpolating 63 data points. The analytic fairing algorithm is one of the existing fairing algorithms. It performs global fairing and uses analytic fairness metrics derived from surface geometry [5]. As the right view shows, the discrete fairing algorithm fairs the original surface and preserves the local shapes of the original surface, while the analytic fairing algorithm removes all of the local shapes. Fig. 3 shows the mean curvature graphs for the fairing results and explains the fairing results well. Because the analytic fairing algorithm performs excessive fairing, the mean curvature graph of the surface faired with it is almost flat. However, the mean curvature graph of the surface faired with the discrete fairing algorithm is smoother than the original mean curvature graph. In addition, the pattern of local shapes of the original mean curvature graph is preserved. The right view shows these results clearly. The reason of the shape preservation comes from the fact that the proposed discrete local fairing algorithm improves fairness at fewer points, which cause only local irregularities.

\section{Conclusions}

We proposed a discrete fairing algorithm. The algorithm performs local and discrete fairing of data points of B-spline surfaces. It fairs the data points and preserves the local shapes of an original surface better than existing global fairing algorithms. This is due to its local and discrete fairing. In addition, the algorithm has less computation time, because it has one free point per a fairing iteration and uses discrete differential geometry.

Therefore, when designers want to preserve the pattern of local shapes of an initial surface after fairing, the proposed algorithm can be adopted and used. There are two future works to be continued. One is to develop another fairness 


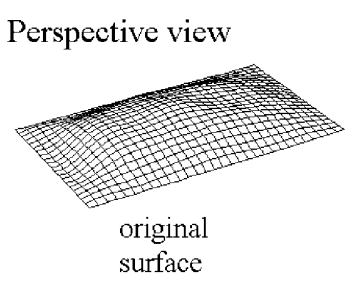

Right view

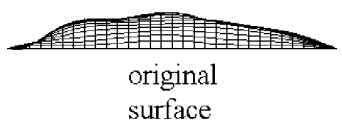

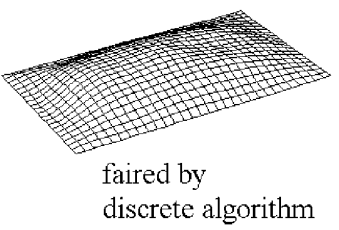

discrete algorithm

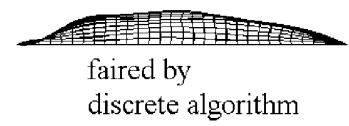

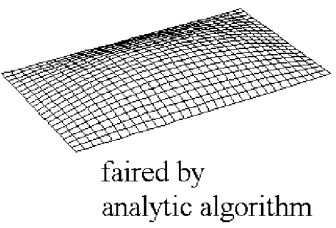

analytic algorithm

Fig. 2. Fairing results with rolling metric

Perspective view

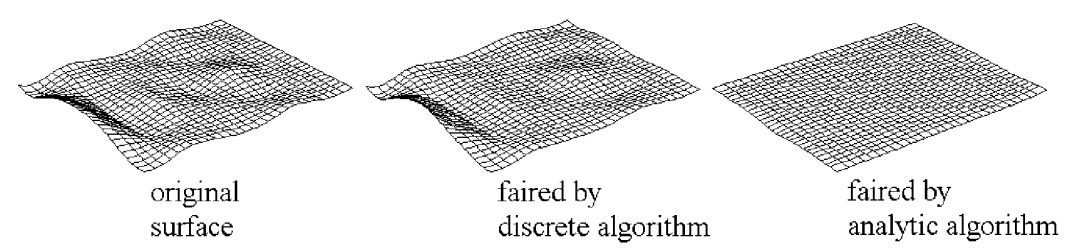

Right view

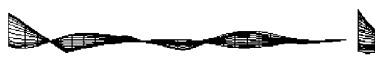

original

surface

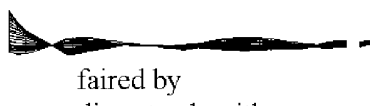

discrete algorithm

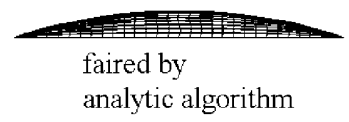

faired by

analytic algorithm

Fig. 3. Mean curvature graphs for the fairing results with rolling metric

metrics and the other is to construct a new surface fairing algorithm, which does not adopt optimizations but uses analytic equations to improve fairness.

\section{References}

1. Choi, B. K.: Surface Modeling for CAD/CAM. Elsevier, Amsterdam Oxford New York Tokyo (1991) 25-29

2. Eck, M. and Jaspert, R.: Automatic Fairing of Point Sets. Designing Fair Curves and Surfaces. Society for Industrial and Applied Mathematics, Philadelphia (1994) 45-60

3. Lott, N. J. and Pullin, D. I.: Method for Fairing B-spline Surfaces. Computer-Aided Design. 10 (1988) 597-604

4. O'Neill, B.: Elementary Differential Geometry. Academic Press (1966) 199-202

5. Rando, T. and Roulier, J.A.: Measures of Fairness for Curves and Surfaces. Designing Fair Curves and Surfaces. Society for Industrial and Applied Mathematics, Philadelphia (1994) 75-122 